\title{
AlGaInAs/InP Based Five \& Three Quantum Wells Mode Locked Laser Diodes: A Comparative Study
}

\author{
Jehan Akbar ${ }^{1,2}$, Muhammad Hanif ${ }^{1}$, Muhammad Azhar Naeem ${ }^{3,}$, Kamran Abid $^{3}$ \\ ${ }^{1}$ Department of Physics, Hazara University Mansehra, \\ KPK, Pakistan \\ ${ }^{2}$ Abdus Salam International Centre for Theoretical Physics, \\ Trieste, Italy \\ ${ }^{3}$ Department of Electrical Engineering, University of the Punjab, \\ Lahore, Pakistan \\ azhar@pu.edu.pk
}

\begin{abstract}
Comparison of performance of semiconductor mode-locked laser diodes fabricated using AlGaInAs/InP material containing 5 and 3 quantum wells (QWs) inside the active region is reported. The simulations and experimental results show that lasers containing five $Q W s$ materials produce larger beam divergence and temporally broader optical pulses. For improvement in the mode-locking of lasers and reducing the far-field pattern, the number of QWs inside the active region was decreased from five to three and a far-field decreasing layer along with a thick spacer layer were introduced in the n-cladding region of epitaxial material. Before growing the material, simulations were carried out to optimise the design. The lower optical confinement factor and higher gain saturation energy of three QWs based mode-locked lasers provide higher average and peak output power, reduced and symmetric far-field pattern, better radio frequency (RF) spectra, shorter optical pulses, and stable optimal modelocking for a wide range of gain current and saturable absorber reverse voltage.
\end{abstract}

Keywords-Quantum well lasers; Laser mode locking; Optical pulses; Optical device fabrication.

\section{INTRODUCTION}

The semiconductor lasers are very important and effective sources of short and stable optical pulses. Short optical pulses from semiconductor lasers have a wide range of scientific and industrial applications, which include optical fiber communication systems, terahertz radiations generation, and investigation of non-linear effects. The semiconductor mode-locked lasers (MLLs) are effective sources of generation of short and high repetition frequency optical pulses with tunable wavelength [1].

The semiconductor MLLs are attractive due to their higher efficiency, lower cost, and shorter cavity lengths. Further, their easy integrability with other devices and ease of fabrication make them very useful [2]. The non-linearities inside the cavity, such as self-phase modulation, tend to broaden the optical pulses and result in an increase in the

Manuscript received 22 January, 2020; accepted 14 August, 2020.

This research was funded by the Project Management Unit, Higher Education Department (KPK, Pakistan) under Grant No. HEREF 144. timing jitter of the lasers output. The non-linearities prevent the emission of shorter optical pulses from semiconductor MLLs [3]. In recent years, monolithic quantum well based mode-locked lasers have shown substantial improvements in the performance in hybrid and passive mode-locking schemes [4]. The results reported in [5], [6] show, that as the number of quantum wells decreases in the active layer of the laser, the pulse width decreases due to the higher saturation energy of gain. The saturation energies of the gain and the absorber section are quite important in determining the range of the stable mode-locking of a MLL. Stability of mode-locking is given by the interplay between the carrier lifetimes, gain saturation, and the saturable absorption in gain section and saturable absorber section of the laser [7]. Moreover, the decrease in optical confinement factor leads to the decrease in RF linewidth due to lower coupling of Amplified spontaneous emission (ASE) to the optical modes [8].

In this manuscript, we report on the MLLs based on an optimized epitaxial structure and also compare the devices fabricated on the novel material structure with the devices based on commercially available material structure. The novel epitaxial structure was optimized for improving the pulse energies, emission stability, timing jitter, and pulse width and chirp.

\section{MATERIAL DESIGN AND DEVICE FABRICATION}

The epitaxial material that is used for the fabrication of lasers was commercially available in $\mathrm{AlGaAs} / \mathrm{InP}$ wafer structure. Details of this material structure can be found in [9].

For improvement in the mode-locking performance of mode-locked lasers, this epitaxial material was modified by reducing the QWs from 5 to 3 and inserting a far-field reduction layer. This leads to a lower differential gain and increase in the mode size [10].

Figure 1 shows the simulation results of optical intensity in the material. These simulations of calculation of intensity profile inside the material were performed using the Laser Technology Integrated Program (LASTIP), which can be 
used to perform 2-D simulations of semiconductor lasers. The results of simulations show that the zero order (fundamental mode) is well confined inside the active region. The results of simulations for the optimized far-field reduction layer of thickness $160 \mathrm{~nm}$ and a spacer layer of thickness $750 \mathrm{~nm}$ are presented in the Fig. 1. Simulation results of laser modes and optical confinement for the material structure reported in [10] demonstrate that if farfield reduction layer is made much thicker, the zero order mode coupling to the far-field reduction layer increases, which leads to the reduction of confinement factor of quantum wells for zero-order mode and extra peaks appear in near-field pattern. The reduction of number of QWs and the insertion of far-field reduction layer in material structure would lead to an increase in beam spot size and maintain fundamental transverse mode.

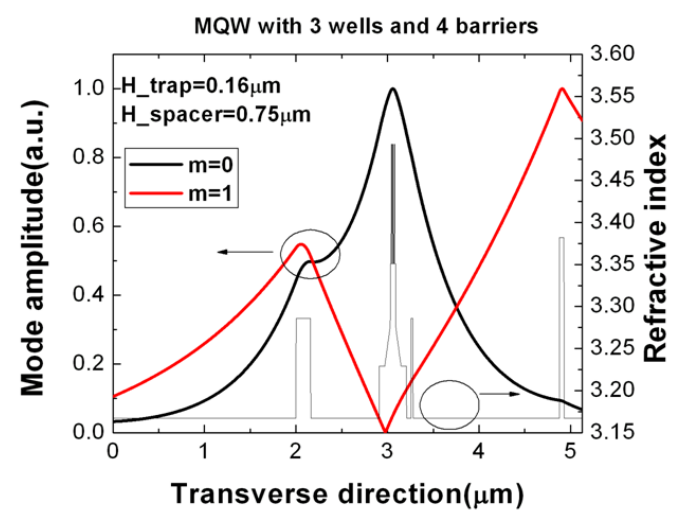

Fig. 1. Simulation results of light intensity profile for fundamental mode (black) and $1^{\text {st }}$ order mode (red) inside the material structure.

In our material design, we decreased QWs to three for decreasing confinement factor of light, and hence differential gain $(\mathrm{dg} / \mathrm{dN})$, which leads to a higher gainsaturation energy $\left(G_{\text {sat }}\right)$. An increase in $G_{\text {sat }}$ leads to reduction in the self-phase modulations inside the gain medium. Further, a far-field reduction layer (FRL) is introduced in the lower (n cladding) of this material which is a weaker waveguide and it stretches the optical modes in the vertical direction, i.e., towards the direction of $\mathrm{n}$ cladding region. This far-field reduction layer is a weaker waveguide and it stretches the optical modes in vertical direction, i.e., towards the direction of $\mathrm{n}$ cladding region. The expansion of near-field leads to reduction in the corresponding far-field pattern [10]. The reduction in divergence angles produces a symmetric far-field pattern, which improves coupling efficiency of the device with a single mode fiber. The farfield reduction layer also suppresses the lasing of higher mode. This suppression of lasing of higher order modes leads to an increase in kink-free output power.

Semiconductor MLLs on both the 5-QWs and optimized 3-QWs epitaxial materials were fabricated using standard semiconductor laser fabrication techniques, such as electron beam lithography, dry-etching, planarization, and metallization. The whole length of the fabricated devices was $1075 \mu \mathrm{m}$, which includes $25 \mu \mathrm{m}$ long saturableabsorber section, $1035 \mu \mathrm{m}$ long gain section, and gap of $15 \mu \mathrm{m}$ width between the two sections as shown in the Fig.3.

For fabrication of the devices, the width and the ridge waveguides height need to be carefully designed to ensure single transverse mode emission from the laser. A commercial mode solver called "BEAMPROP" was used for 3-D simulations for designing a ridge waveguide, which supports only fundamental (zero-order) transverse mode in the device.

To find optimal width of the ridge for single transverse mode operation, the simulated mode index for the zeroorder, $1^{\text {st }}$ order, and $2^{\text {nd }}$ order Transverse Electric (TE) modes for different waveguide width and ridge height of $1.92 \mu \mathrm{m}$ is shown in Fig. 2. It is clear from the Fig. 2 that waveguides below the ridge-width of $0.6 \mu \mathrm{m}$ do not support any modes. The cut-off of the fundamental (zero-order) transverse mode is at $2 \mu \mathrm{m}$ wide waveguide.

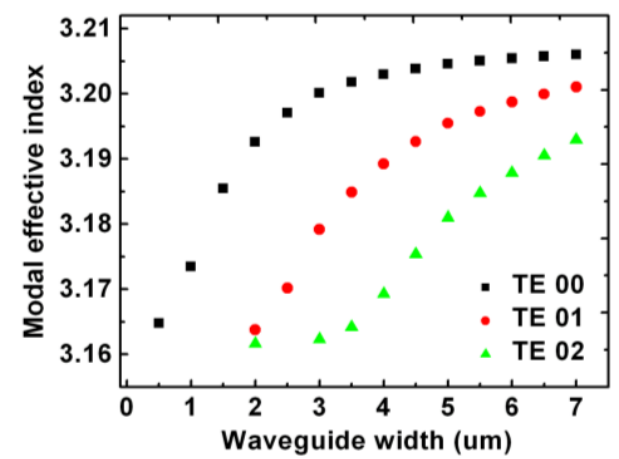

Fig. 2. Simulation results for different widths of the ridge waveguides.

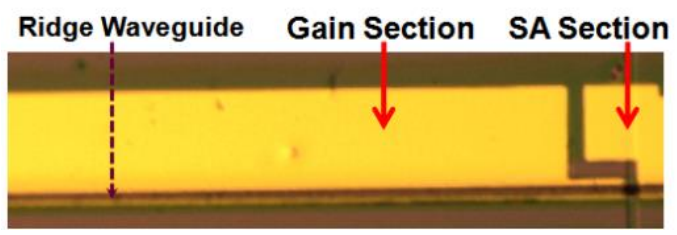

Fig. 3. Schematic of the fabricated mode-locked laser.

After fabrication, these devices were cleaved into individual bars and these cleaved bars were placed on a temperature controlled copper heat sink for characterization.

\section{DEVICES CHARACTERIZATION}

The results obtained both from 5-QWs and 3-QWs material based devices are presented in the following subsections.

\section{A. Output Power Measurements}

The light-current (L-I) curve gives information about the maximum average output-power $(\mathrm{P})$, threshold current $\left(\mathrm{I}_{\mathrm{th}}\right)$, and slope efficiency $(\mathrm{y})$ of the lasers.

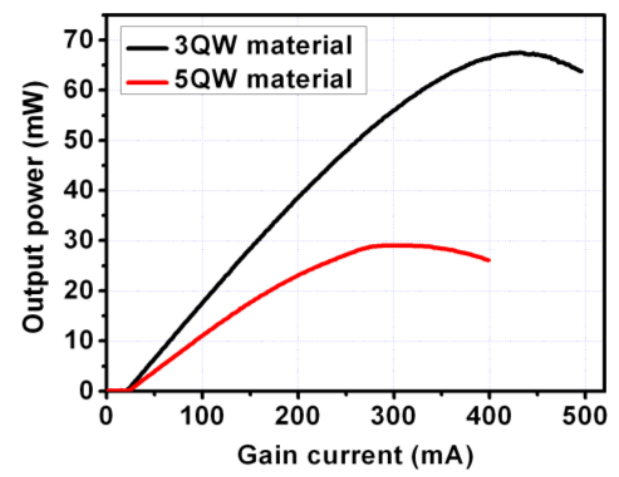

Fig. 4. Comparison of L-I curves measured from 3-QWs and 5-QWs devices. 
The LI graphs of simple ridge waveguide Fabry Perot (FP) lasers fabricated using 5-QWs and 3-QWs epitaxial structures are shown in Fig. 4. The threshold current for both kinds of devices is nearly the same, i.e., $25 \mathrm{~mA}$. Moreover, for the 3-QWs laser, the slope efficiency is about $0.21 \mathrm{~W} / \mathrm{A}$, while that for the 5-QWs based device is 0.14 W/A. An output power of around $68 \mathrm{~mW}$ is obtained from 3-QWs devices, while only $28 \mathrm{~mW}$ are obtained from 5-QWs material based devices.

\section{B. Mode-Locking Characterizations}

For measuring the mode-locking characteristics, the gain section of the device was forward biased, while the saturable-absorber section was reverse biased. Light generated and emitted from the fabricated lasers was coupled via a single-mode lensed optical-fiber, which is linked to the characterization equipment via Faraday isolator. The isolator was used for preventing back reflections into the laser cavity.

The mode-locking characterization of the devices was carried out in 3 main steps, i.e., optical spectrum measurement, RF spectrum measurement, and the measurement of $2^{\text {nd }}$ harmonic generation (SHG) intensity autocorrelation traces.

For measuring the optical spectra, the light emitted from the devices was analysed using an optical spectrum analyser (OSA). The optical spectrum obtained from the 3-QWs and 5-QWs lasers under the optimal mode-locked operation is given in Fig. 5. These spectra were measured using optical spectrum analyser with resolution band-width (BW) of $0.06 \mathrm{~nm}$.

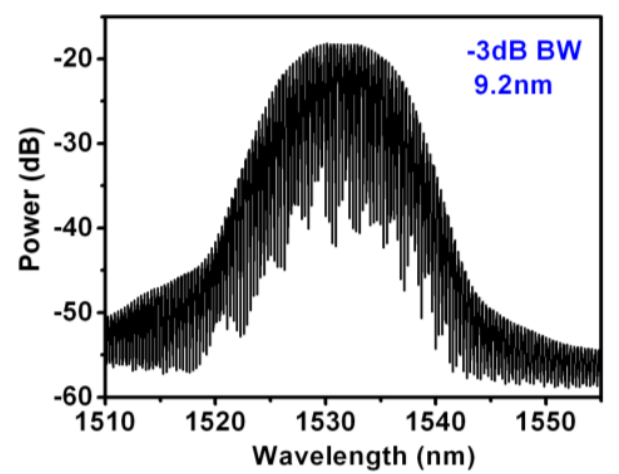

(a)

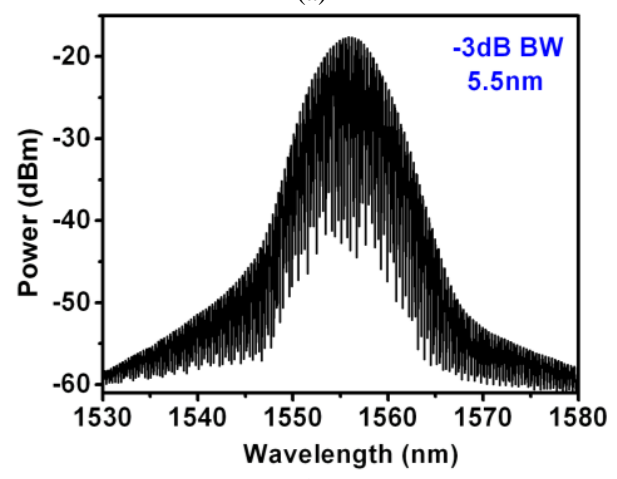

(b)

Fig. 5. Measured optical spectra of the (a) 3-QWs MLL at $I_{\text {gain }} 60 \mathrm{~mA}$ and $\mathrm{V}_{\mathrm{SA}}-3 \mathrm{~V}$, and (b) 5-QWs MLL at $\mathrm{I}_{\text {gain }} 72 \mathrm{~mA}$ and $\mathrm{V}_{\mathrm{SA}}-3 \mathrm{~V}$.

The measured optical spectrum of 3-QWs based MLLs gives a $3 \mathrm{~dB}$ bandwidth of $9.2 \mathrm{~nm}$ with wavelength $(\lambda)$ centered at $1532 \mathrm{~nm}$. The optical spectrum of 5-QWs based
MLLs gives $3 \mathrm{~dB}$ bandwidth of $5.5 \mathrm{~nm}$ with wavelength centered at $1556 \mathrm{~nm}$. The $3 \mathrm{~dB}$ bandwidth of a laser shows the number of phase locked modes that is inversely proportional to the temporal output pulse-width. This means, the 3-QWs based MLLs will emit shorter optical pulses [11]. Further, for both MLLs, the optical spectra are very symmetrical and the mode spacing is about $0.32 \mathrm{~nm}$, which gives around $40 \mathrm{GHz}$ repetition rate. The mode-locking of the devices can be confirmed by measuring the radio frequency (RF) spectrum, which also shows the repetition frequency of the MLLs. Similarly, the RF spectrum presented in Fig. 6 shows, that for the 3-QWs MLLs, the fundamental mode-locking frequency is $39.75 \mathrm{GHz}$, while that for 5-QWs based MLLs, it is $39.65 \mathrm{GHz}$, respectively. The RF peak obtained from the 3-QWs based MLLs is around is $40 \mathrm{~dB}$ above the noise floor, while that of 5-QWs based MLLs is $35 \mathrm{~dB}$ above the noise floor. For the 3-QWs MLLs, the $3 \mathrm{~dB}$ line-width of RF spectrum (with Lorentzian fit) is $130 \mathrm{kHz}$, while for 5-QWs based devices, its value increased to around $900 \mathrm{kHz}$. The narrower RF spectra obtained from 3-QWs based devices is due to the lower amplified spontaneous emission (ASE) noise, which is due to spontaneous coupling co-efficient loss [12].

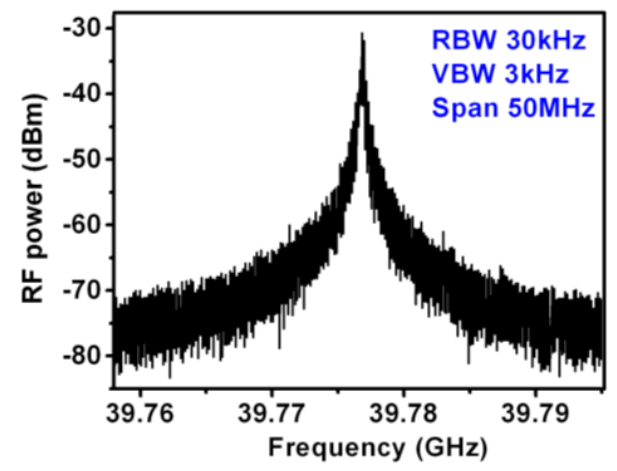

(a)

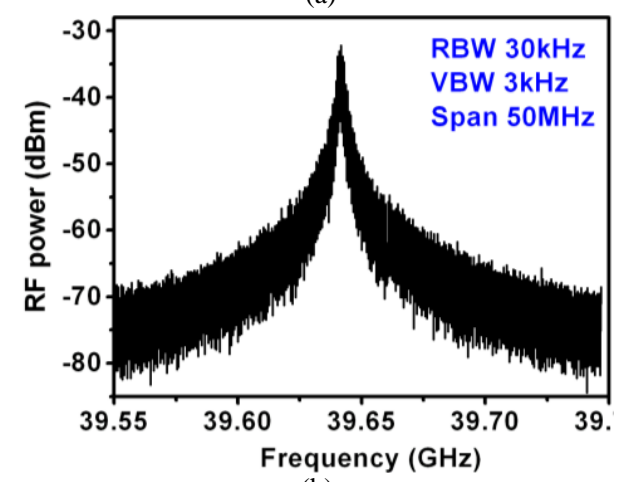

(b)

Fig. 6. Measured RF spectra of the (a) 3-QWs MLL at $I_{\text {gain }} 60 \mathrm{~mA}$ and $\mathrm{V}_{\mathrm{SA}}$ - $3 \mathrm{~V}$ and (b) 5-QWs MLL at $\mathrm{I}_{\text {gain }} 72 \mathrm{~mA}$ and $\mathrm{V}_{\mathrm{SA}}-3 \mathrm{~V}$.

A common technique to measure sub-10 ps optical pulses is using the SHG intensity autocorrelation method. This method is based on the combination of an optical pulse with a time-delayed replica of the same pulse inside a non-linear medium.

The optical pulses emitted from the MLLs were coupled to second harmonic generation (SHG) intensity autocorrelator via single mode optical fiber, polarization controller, an optical isolator, and an erbium doped fiber amplifier (EDFA).

Figure 7 shows measured single (isolated) optical pulse 
emitted by the 3-QWs and 5-QWs based semiconductor MLLs, respectively. The best fitting for a single pulse obtained from auto-correlation traces was Sech2 fitting. The Full Width Half Maximum (FWHM) obtained for a single output pulse obtained from 3-QWs semiconductor MLL was found to be around $1.40 \mathrm{ps}$, which gives to $0.9 \mathrm{ps}$ pulse width. The FWHM of the auto-correlation (AC) trace obtained from 5-QWs based MLL was $2.08 \mathrm{ps,} \mathrm{which}$ deconvolves to $1.3 \mathrm{ps}$ pulse duration. The shorter optical pulses emitted by 3-QWs MLLs than the 5-QWs MLLs are because of the reduced number of quantum wells inside the material active region, which leads to reduction in the material differential gain and gives a higher value of gain saturation energy. The higher gain saturation energy reduces pulse broadening in the gain medium [13].

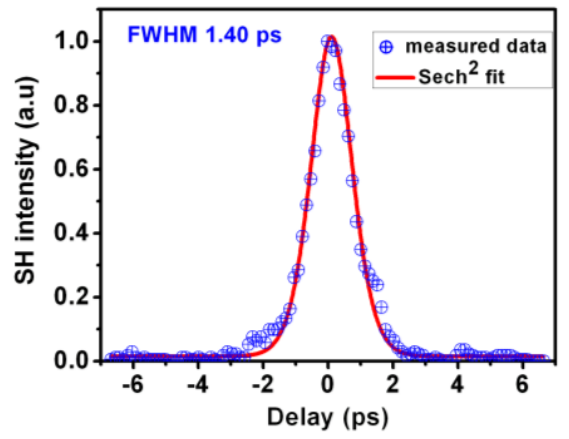

(a)

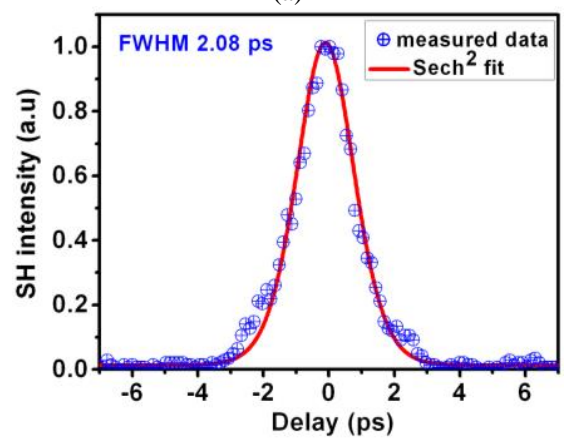

(b)

Fig. 7. Measured single optical pulse of the (a) 3-QWs MLL at $I_{\text {gain }} 60 \mathrm{~mA}$ and $\mathrm{V}_{\mathrm{SA}}-3 \mathrm{~V}$ and (b) 5-QWs MLL at $\mathrm{I}_{\text {gain }} 72 \mathrm{~mA}$ and $\mathrm{V}_{\mathrm{SA}}-3 \mathrm{~V}$.

The far-field pattern of a laser shows the spread of laser mode at different angles, and therefore it is very important in finding coupling-efficiency ( $\mathrm{y}$ ) of the device and the optical fiber. Figure 8 shows the BEAMPROP simulation results of the far-field pattern for 3-QWs and 5-QWs lasers, respectively.

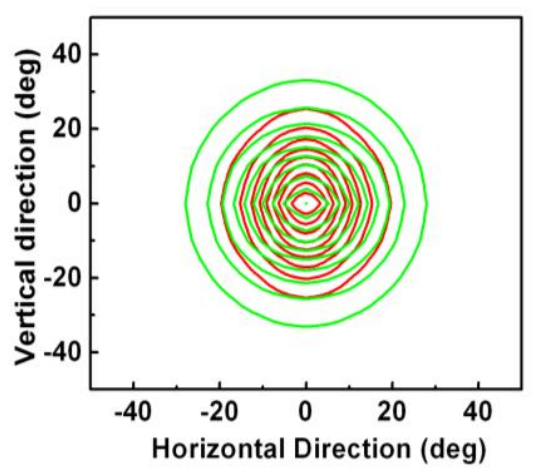

Fig. 8. Simulation results: Far-field pattern of 3-QWs (red curve) and 5QWs (green curve) lasers. Each circular line around the centre denotes a $10 \%$ decrease in the optical intensity.
The simulation results show that the far-field of the 3QWs laser (red curve) in the horizontal direction (x-axis) is $22^{\circ}$, and in vertical direction (y-axis), the divergence is $28^{\circ}$. Similarly, the far-field of the 5-QWs laser (green curve) is $29^{\circ}$ in the horizontal and $34^{\circ}$ in vertical directions.

The far-field pattern of both kinds of devices, i.e., 3-QWs and 5-QWs lasers, was experimentally measured after the fabrication, which is shown in Fig. 9.

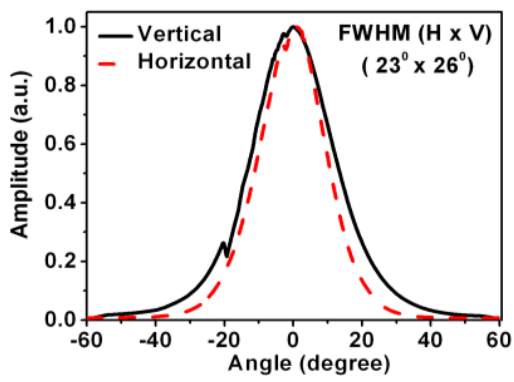

(a)

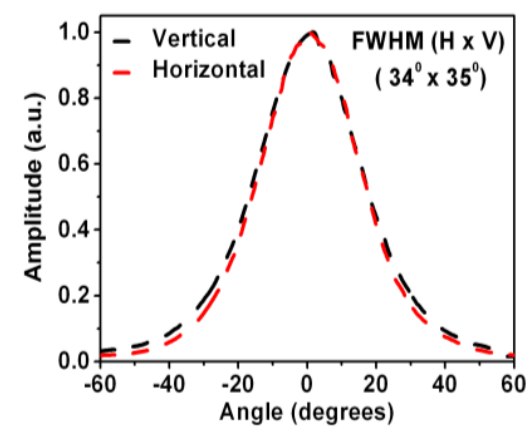

(b)

Fig. 9. Measured far-field of the (a) 3-QWs and (b) 5-QWs mode-locked lasers.

Figure 9 shows that the divergence angle of $3-\mathrm{QWs}$ device is $23^{\circ}$ in horizontal direction and $26^{\circ}$ in the vertical direction. Similarly, the far-field of the 5-QWs devices in horizontal (x) direction is around $34^{\circ}$, while $35^{\circ}$ in the vertical (y) direction. For the 3-QWs material based lasers, the reduction in divergence angles is because of introduction of far-field reduction epitaxial layer in the material structure. The intensity profile of the 3-QWs based lasers is shown in Fig. 9. It is clear from the Fig. 9 that the far-field pattern is single moded and symmetric.

The simulations (Fig. 8) and the experimentally measured (Fig. 9) results of far-field pattern are not very much consistent with each other, which might be due fabrication flaws (geometrical) in the shape of the fabricated ridge waveguides.

To compare the mode-locking performance of lasers fabricated using 3-QWs and 5-QWs materials, mode-locked lasers from both these materials were fabricated using similar fabrication procedure. After fabrication, the modelocking maps of both these devices were made under different bias conditions. Depending upon the injection current to the gain sections and reverse voltage of SA sections, the devices operate in different pulsed regimes as shown in Fig. 10. The red color indicates the optimal modelocking, dark red indicates $\mathrm{Q}$ switching, orange indicates $75 \%$ mode-locking, yellow indicates $50 \%$ mode-locking, while blue color shows Continuous wave $(\mathrm{CW})$ emission 
(no mode-locking). Different mode-locking regimes are categorized on the basis of optical spectrum, the Radio Frequency spectra noise ratio, and the extension ratio (ER) of second harmonic generation (SHG) auto-correlator optical pulses. Additionally, RF spectrum for low frequencies (i.e., $1 \mathrm{GHz}-10 \mathrm{GHz}$ ) is also experimentally measured for identification of Q-switching. For each absorber reverse voltage $\left(\mathrm{V}_{\mathrm{abs}}\right)$, an increase in the gain injection current $\left(\mathrm{I}_{\text {gain }}\right)$ leads to increase in optical pulse width and ultimately causes to switch the laser to from pulsed to $\mathrm{CW}$ regime.

Our results indicate that the pulse energy is rapidly increasing with an increase in injection current of the gain section. As the pulse energy increases, the required SA voltage $\left(-\mathrm{V}_{\mathrm{SA}}\right)$ is also increasing for increasing the SA absorption, and therefore provides maximum pulse reduction, which is a basic requirement for stable and optimal mode-locking operation of lasers [4]. The interplay between gain saturation and carriers' lifetime inside gain and SA sections determines the optimal mode-locking in the devices [14]. One of the most important factors which determines mode-locking stability of lasers is the energygaps detuning between the gain and SA sections. As reported in [15], stable and optimal mode-locking is achieved when the gain and SA sections are aligned in spectrum. The total length of absorber section is also a main factor for determining the range of optimal and stable modelocking of the devices. If the SA length is very short, then it is not enough for triggering the mode-locking in the devices, while too long SA section leads to increased optical losses, which in turn decreases power in the cavity [15]. For an optimized absorber section length, optimal and stable modelocking for broader range of SA bias voltages and gain injection currents occurs. As presented in Fig. 10, for the 5QWs lasers, stable and optimal mode-locking is obtained for $\mathrm{I}_{\text {gain }}$ ranging from $70 \mathrm{~mA}$ to $108 \mathrm{~mA}$ and absorber reverse voltages - from $2.4 \mathrm{~V}$ to $3.5 \mathrm{~V}$. Similarly, Fig. 10 also shows, that for the 3-QWs devices, optimal and stable mode-locking is obtained for $I_{\text {gain }}$ from $60 \mathrm{~mA}$ to $112 \mathrm{~mA}$ and SA section reverse voltages - from $1.8 \mathrm{~V}$ to $3.7 \mathrm{~V}$.

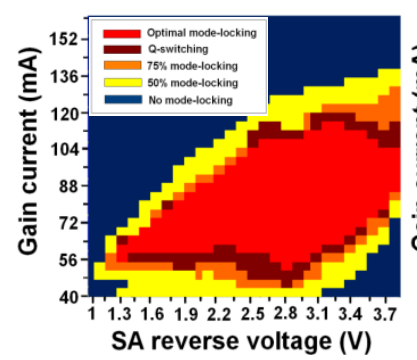

(a)

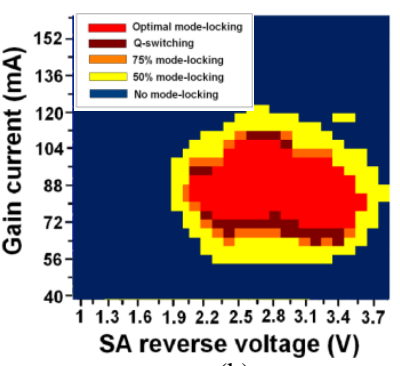

(b)
Fig. 10. Mode-locking maps showing operation of the (a) 3-QWs and (b) 5-QWs MLLs for different gain injection currents and absorber reverse voltages.

Saturation energies of gain section and the absorber section are very important for determining the range of optimal and stable mode-locking of lasers. The gain saturation energy is very strongly dependant on the number of QW in the active region. It has a larger value for the reduced number of QWs because of the lower differential gain [3]. The larger saturation energy of the gain section of 3-QWs devices, as compared to 5-QWs devices, results in lesser optical pulse-broadening, which ultimately leads to larger region of optimal and stable mode-locking as presented in Fig. 10. On the other hand, there is a stronger pulse broadening in the gain section of 5-QWs based lasers, and therefore pulse broadening of the gain section cannot be compensated by the absorber over a larger region (wide range of bias conditions).

\section{CONCLUSIONS}

We have shown that mode-locking performance has been improved by the optimization of the epitaxial structure of the laser material. Reducing the number of QWs inside the active region and inserting a far-field, reduction increase the mode size and increase the gain saturation energy. Modelocking operation of 3-QWs and 5-QWs MLLs laser has been assessed. Reducing the number of QWs from 5 to 3 leads to larger $3 \mathrm{~dB}$ bandwidth of optical spectrum (increased from $5.5 \mathrm{~nm}$ to $9.2 \mathrm{~nm}$ ), narrower RF spectrum (decreased from $900 \mathrm{kHz}$ to $130 \mathrm{kHz}$ ), and shorter optical pulses (decreased from $1.3 \mathrm{ps}$ to $0.9 \mathrm{ps}$ ). Furthermore, the divergence angles of the 3-QWs material based device are $23^{\circ}$ and $26^{\circ}$ in the horizontal and vertical directions, respectively, while the divergence angles of the device based on 5-QWs material are $34^{\circ}$ and $35^{\circ}$ in the horizontal and vertical directions. This improvement in the divergence angles leads to better coupling efficiency with optical fibers.

\section{ACKNOWLEDGMENT}

The authors wish to thank the staff of James Watt Nanofabrication Center at Univerity of Glasgow, UK for their technical assistance and Higher Education Commission of Pakistan, Higher Education Department, and Government of KPK for their support under HEREF project.

\section{CONFLICTS OF INTEREST}

The authors declare that they have no conflicts of interest.

\section{REFERENCES}

[1] D. J. Derickson, R. J. Helkey, A. Mar, J. R. Karin, J. G. Wasserbauer and J. E. Bowers, "Short pulse generation using multisegment mode locked semiconductor lasers", IEEE J. Quantum Electronics, vol. 28 , no. 10, pp. 2186-2202, 1992. DOI: 10.1109/3.159527.

[2] E. A. Avrutin, J. H. Marsh, and E. L. Portnoi, "Monolithic and multigigahertz mode-locked semiconductor lasers: Construction, experiments, models and applications", IEEE Proceedings Optoelectronics, vol. 147, no. 4, 2000. DOI: 10.1049/ipopt:20000282.

[3] K. Yvind et al., "Design and evaluation of mode-locked semiconductor lasers for low noise and high stability", in Proc. of Proc. SPIE 5825, Opto-Ireland 2005: Optoelectronics, Photonic Devices, and Optical Networks, 2005. DOI: 10.1117/12.611341.

[4] K. A. Williams, M. J. Thompson, and I. H. White, "Long-wavelength monolitic mode-locked diode lasers", New J. Phys., vol. 6, pp. 1-30, 2004. DOI: 10.1088/1367-2630/6/1/179.

[5] Ch. Weber, A. Klehr, A. Knigge, and S. Breuer, "Picosecond pulse generation and pulse train stability of a monolithic passively modelocked semiconductor quantum-well laser at $1070 \mathrm{~nm}$ ", IEEE Journal of Quantum Electronics, vol. 54, no. 3, pp. 1-9, 2018. DOI: 10.1109/JQE.2018.2832288.

[6] K. Yvind, D. Larsson, L. J. Christiansen, C. Angelo, L. K. Oxenlwe, J. Mrk, D. Birkedal, J. M. Hvam, and J. Hanberg, "Low jitter and high power $40 \mathrm{GHz}$ all active mode locked lasers", IEEE Photon. Technol. Lett., vol. 16, no. 4, pp. 975-977, 2004. DOI: 10.1109/LPT.2004.824634.

[7] S.-H. Wei et al., "Spectral dynamical behavior in two-section, quantum well, mode-locked laser at $1.064 \mu \mathrm{m}$ ", Chinese Physics B, vol. 26, no. 7, 2017. DOI: 10.1088/1674-1056/26/7/074208. 
[8] F. Lelarge, B. Dagens, J. Renaudier, R. Brenot, A. Accard, F. van Dijk, D. Make, O. Le Gouezigou, J.-G. Provost, F. Poingt, J Landreau, O. Drisse, E. Derouin, B. Rousseau, F. Pommereau, and G.-H. Duan, "Recent advances on InAs/InP quantum dash based semiconductor lasers and optical amplifiers operating at $1.55 \mu \mathrm{m}$ ", IEEE J. Sel. Top. Quantum. Electron., vol. 13, no. 1, pp. 111-124, 2007. DOI: 10.1109/JSTQE.2006.887154.

[9] J. Akbar, L. Hou, M. Haji, M. J. Strain, J. H. Marsh, A. C. Bryce, and A. E. Kelly, "High power $(130 \mathrm{~mW}) 40 \mathrm{GHz} 1.55 \mu \mathrm{m}$ mode-locked distributed Bragg reflector lasers with integrated optical amplifiers", Opt. Lett., vol. 37, no. 3, pp. 344-346, 2012. DOI: 10.1364/OL.37.000344.

[10] B. Qiu, S. D. McDougall, X. Liu, G. Bacchin, and J. H. Marsh, "Design and fabrication of low beam divergence and high kink-free power lasers", IEEE Journal of Quantum Electronics, vol. 41, no. 9, pp. 1124-1130, 2005. DOI: 10.1109/JQE.2005.853359.
[11] G. P. Agrawal and N. K. Dutta, Long-Wavelength Semiconductor Lasers. Van Nostrand Reinhold: New York, 1986. DOI: 10.1007/97894-011-6994-3.

[12] F. Kefelian et al., "RF linewidth in monolithic passively mode-locked semiconductor lasers", IEEE Photonics Technology Letters, vol. 20, no. 16, pp. 1405-1407, 2008. DOI: 10.1109/LPT.2008.926834.

[13] D. J. Derickson et al., "Short pulse generation using multisegment mode-locked semiconductor lasers", IEEE J. of Quantum Electronics, vol. 28, no. 10, pp. 2186-2202, 1992. DOI: 10.1109/3.159527.

[14] J. Javaloyes and S. Balle, "Mode-locking in semiconductor FabryPérot lasers", IEEE J. of Quantum Electronics, vol. 46, no. 7, pp. 1023-1030, 2010. DOI: 10.1109/JQE.2010.2042792.

[15] D. Kunimatsu, S. Arahira, and Y. Ogawa, "Passively mode-locked laser diodes with bandgap-wavelength detuned saturable absorbers", IEEE Photonics Technology Letters, vol. 11, no. 11, pp. 1363-1365, 1999. DOI: 10.1109/68.803046.

This article is an open access article distributed under the terms and conditions of the Creative Commons Attribution 4.0 (CC BY 4.0) license (http://creativecommons.org/licenses/by/4.0/). 